\title{
Behavioral, affective, and cognitive alterations induced by individual and combined environmental stressors in rats
}

\author{
Francisco Diego Rabelo-da-Ponte, ${ }^{1}$ Jessica Maria Pessoa Gomes, ${ }^{1}$ Nathércia Lima Torres, ${ }^{1}$ \\ João llo Coelho Barbosa, ${ }^{1}$ Geanne Matos de Andrade, ${ }^{2}$ Danielle Macedo, ${ }^{3,4}$ Bruno Ceppi $^{2}$ (iD \\ ${ }^{1}$ Departamento de Psicologia, Universidade Federal do Ceará (UFC), Fortaleza, CE, Brazil. ${ }^{2}$ Laboratório de Neurociências e Comportamento, \\ Departamento de Fisiologia e Farmacologia, UFC, Fortaleza, CE, Brazil. ${ }^{3}$ Laboratório de Neurofarmacologia, Departamento de Fisiologia e \\ Farmacologia, Núcleo de Pesquisa e Desenvolvimento de Medicamentos, UFC, Fortaleza, CE, Brazil. ${ }^{4}$ Instituto Nacional de Ciência e \\ Tecnologia Translacional em Medicina (INCT-TM), Conselho Nacional de Desenvolvimento Científico e Tecnológico (CNPq), Ribeirão Preto, \\ $S P$, Brazil.
}

\begin{abstract}
Objective: To evaluate whether exposing rats to individual or combined environmental stressors triggers endophenotypes related to mood and anxiety disorders, and whether this effect depends on the nature of the behavior (i.e., innate or learned).

Methods: We conducted a three-phase experimental protocol. In phase I (baseline), animals subjected to mixed schedule of reinforcement were trained to press a lever with a fixed interval of 1 minute and a limited hold of 3 seconds. On the last day of phase I, an open-field test was performed and the animals were divided into four experimental groups ( $n=8 /$ group). In phase II (repeated stress), each group was exposed to either hot air blast (HAB), paradoxical sleep deprivation (PSD) or both (HAB + PSD group) on alternate days over a 10-day period. Control group animals were not exposed to stressors. In phase III (post-stress evaluation), behavior was analyzed on the first (short-term effects), third (mid-term effects), and fifth (long-term effects) days after repeated stress.

Results: The PSD group presented operant hyperactivity, the HAB group presented spontaneous hypoactivity and anxiety, and the HAB + PSD group presented spontaneous hyperactivity, operant hypoactivity, impulsivity, loss of interest, and cognitive impairment.

Conclusion: A combination of environmental stressors (HAB and PSD) may induce endophenotypes related to bipolar disorder.
\end{abstract}

Keywords: Animal model; combined stressors; paradoxical sleep deprivation; hot air blast; bipolar disorder

\section{Introduction}

Psychiatric disorders are a serious health problem. Currently, of the 20 main causes of disability, six are psychiatric disorders, with depressive disorder accounting for $40.5 \%$ of disability-adjusted life years, anxiety for $14.6 \%$, illicit drug use for $10.9 \%$, schizophrenia for $7.4 \%$, and bipolar disorder for $7 \%{ }^{1}$

Anxiety, mood disorders, substance abuse and schizophrenia are stress-related disorders. ${ }^{2,3}$ Stress can induce a wide range of alterations in an organism, depending on frequency, magnitude, duration, type, context, neurodevelopmental stage, sex, and genetic predisposition. ${ }^{2}$

Animal models are important tools for investigating the effects of stress, such as stress-induced epigenetic alterations. Based on animal models, new therapeutic targets for psychiatric disorders may be identified. ${ }^{3}$ To improve their translational value, animal models should

Correspondence: Bruno Ceppi, Departamento de Fisiologia e Farmacologia, Faculdade de Medicina, Universidade Federal do Ceará, Rua Coronel Nunes de Melo, 1127, Rodolfo Teófilo, CEP 60430-275, Fortaleza, CE, Brazil.

E-mail: bruno.ceppi@gmail.com

Submitted Jan 08 2018, accepted Aug 01 2018, Epub Mar 142019. be able to simulate some aspects of stress response and replicate the natural history of the disease. For instance, stress may be induced by exposure to forced swimming, food-deprivation, neonatal isolation, predation, day-night light change or noise. ${ }^{4}$ Despite the variety of stresses, some reports suggest that using different types of chronic, unpredictable, and inescapable stressors with high ecological validity to improve the translational validity of preclinical models of stress-related disorders, such as depression. ${ }^{5}$

Therefore, despite the importance of environmental stress in the early manifestation, increased severity, and shorter inter-episode intervals of psychiatric disorders, ${ }^{6}$ the translational value of these animal models needs improvement. To this end, the inclusion of operant behavior and the manipulation of ecologically relevant stimuli, namely an inescapable aversive stimulus and sleep deprivation, seems to be an important alternative to

How to cite this article: Rabelo-da-Ponte FD, Pessoa Gomes JM, Torres NL, Barbosa JIC, de Andrade GM, Macedo D, et al. Behavioral, affective, and cognitive alterations induced by individual and combined environmental stressors in rats. Braz J Psychiatry. 2019;41:289-296. http://dx.doi.org/10.1590/1516-4446-2018-0009 
ethopharmacological models. ${ }^{7}$ It should be pointed out that a number of animal models lack of external validity and do not reproduce features of the stress response observed in clinical settings. ${ }^{3}$ Reinforcement schedules have been investigated over the last 6 decades. For instance, individual schedules can be arranged according to ratio, interval, or interresponse time, while combined schedules involve a combination of at least two single schedules. Hence, a behavioral account of reinforcement schedules provides understanding of an organism's performance in terms of specific environment-behavior relationships. ${ }^{8}$

Changes in locomotor activity patterns, the endocrine system and circadian rhythms, including irregular sleepwake cycle, are common features observed in psychiatric disorders. ${ }^{9}$ In addition, alterations in circadian rhythm are known to interact with uncontrollable and inescapable stressful events in new mood disorder episodes. ${ }^{10}$ Learned helplessness, a typical behavior in both humans and animals, is characterized by difficulties in learning new behaviors, decreased sensitivity to new contingencies, and cognitive impairment after exposure to uncontrollable aversive stimuli. ${ }^{11}$

Therefore, based on the fragility of the available animal models for studying mood disorders, we decided to study the interactions between spontaneous/innate and operant/learned behaviors triggered by repeated exposure to either sleep deprivation, inescapable stressful stimuli or a combination of the two. To do this, we used two animal models: paradoxical sleep deprivation (PSD) using the modified multiple platform method and inescapable exposure to hot air blast (HAB). ${ }^{12,13}$ We hypothesized that exposure to individual or combined environmental stressors may trigger endophenotypes related to mood and anxiety disorders, depending on the innate or learned nature of the behavior.

\section{Methods}

\section{Animals and apparatus}

Adult male Wistar rats $(\mathrm{n}=32)$ weighing $300-325 \mathrm{~g}$ were used. The animals were born in the Universidade Federal do Ceará's animal house (Fortaleza, state of Ceará, Brazil). Two rats were housed in each cage and kept in auditory and visual contact under controlled lighting (12 hour light- dark cycle; lights on at 7 a.m.) and temperature $\left(22-23^{\circ} \mathrm{C}\right)$ conditions. Animals from the same group were kept together to avoid behavior contagion from exposure to different stressors. ${ }^{14}$ All experiments were carried out during the light phase of the light-dark cycle.

The animals were randomly divided into four experimental groups that were weighed weekly. The protocols caused no harmful physical modifications to the animals. We used operant conditioning chambers (Insight Ltda., Ribeirão Preto, Brazil), a hair dryer (1,200 W, 85 dB), a digital thermometer, and a sleep deprivation apparatus. The experimental procedures were in accordance with Brazilian legislation on the care and maintenance of experimental animals. The study was approved by the local ethics committee for animal experimentation (protocol 96/13).

\section{Experimental design}

The study was divided into three sequential phases, as follows: phase I or baseline, consisted of pre-stress evaluation; phase II or the repeated stress phase, consisted of exposure to two types of stresses, isolated or combined; and phase III or follow-up, consisted of post-stress evaluation. Figure 1 presents an overview of the experimental design.

At baseline (phase I), reinforcement and open field tests were used to determine learned and innate behaviors, respectively. Operant performance was used as a stability criterion and regarded as an endpoint of this phase. The animals were then underwent an open-field test to facilitate their separation into experimental and control groups. In other words, to balance the experimental groups, animals with strong or weak exploratory drives were allocated in equal proportions to each experimental group.

Phase II consisted of manipulating two environmental stressors, HAB and PSD, on alternate days over 10 days (repeated stress exposure). The HAB group $(n=8)$ was exposed to inescapable HAB five times on alternate days. The PSD group $(n=8)$ was deprived of sleep five times on alternate days. The HAB + PSD group $(n=8)$ was exposed to five sessions of $H A B$ intercalated with five sessions of PSD. The control group $(n=8)$ was not exposed to any

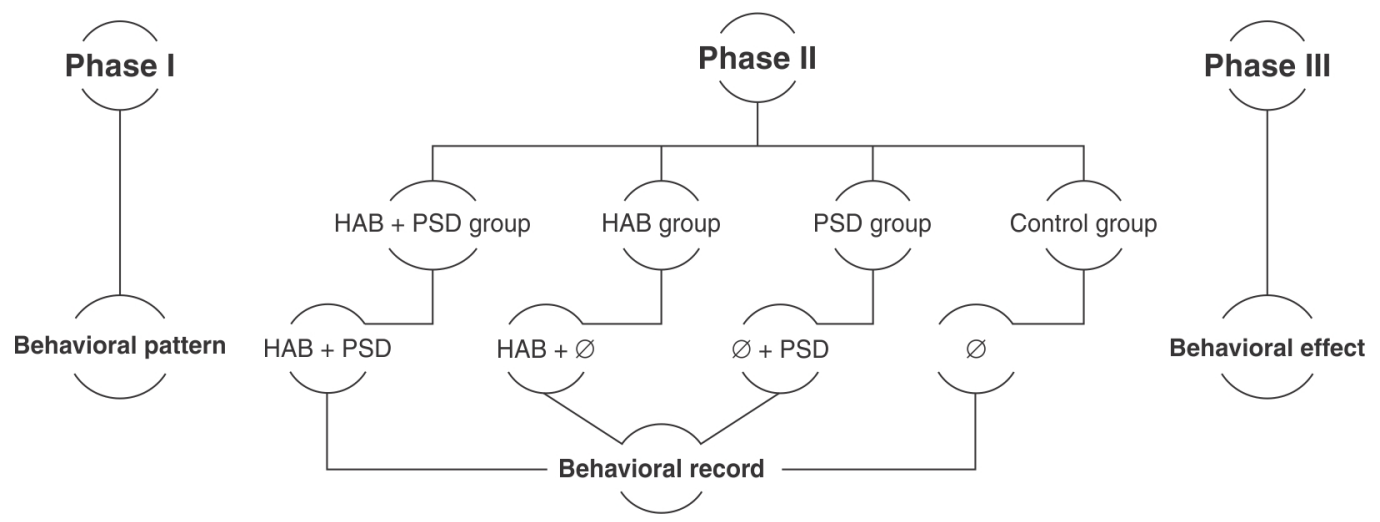

Figure 1 Overview of the experimental design. $\mathrm{HAB}=$ hot air blast; PSD = paradoxical sleep deprivation. 
stressors. The rats were submitted to daily reinforcement sessions.

In phase III we analyzed the animals' short-term ( 1 day after repeated stress exposure), mid-term (3 days after repeated stress exposure), and long-term behavior ( 5 days after repeated stress exposure) to verify changes in spontaneous behavior (through the open field test), goal-directed behavior, and consummatory behavior (through the reinforcement procedure).

\section{Behavioral tests}

\section{Open-field test}

Spontaneous behavior was evaluated with the open-field test. The apparatus was an acrylic arena with an inner dimension of $50 \times 50 \mathrm{~cm}$ and a floor divided into nine equal squares. Each test lasted 5 minutes. The frequency of crossing and entering into the central zone were recorded. A careful cleaning of the apparatus was performed between animals, with $20 \%(\mathrm{v} / \mathrm{v})$ ethanol solution. XPloRat software was used for video recording. ${ }^{15}$

\section{Reinforcement procedure}

Every animal was trained to learn a lever-press response (LR) during the reinforcement procedure. They were then gradually exposed to a mixed schedule of reinforcement with a fixed interval of 1 minute and a limited hold of 3 seconds (mix FI 1' LH3'). In other words, the rat only received a drop of water with a LR before $1 \mathrm{~min}$ had expired, which remained available for 3 seconds. ${ }^{8}$ The endpoint of phase I was the achievement of a scallop pattern in cumulative LR records, i.e., at least $75 \%$ of the total responses emitted in the last 30 seconds of each fixed interval. A total of four behavioral parameters were determined after the reinforcement procedure: i) frequency of total LR (f[LRt])during the session; ii) frequency of received reward $\left(\mathrm{f}\left[\mathrm{S}^{+}\right]\right)$; iii) optimization index $\left(\mathrm{f}\left[\mathrm{S}^{+}\right]\right.$) divided by LR frequency in the last 30 seconds of each fixed interval during the session; iv) scallop pattern, i.e., the percentage of LR in the last 30 seconds of each fixed interval in relation to $f(L R t)$. In summary, the behavioral tests assessed impulsivity/anxiety, locomotor activity, procedural memory, motivation, and behavioral efficiency (Table 1).

\section{Hot air blast}

$\mathrm{HAB}$ exposure followed methods described elsewhere. ${ }^{16}$ Additionally, the operant conditioning chamber's internal temperature was controlled to $\leqslant 49 \pm 0.5{ }^{\circ} \mathrm{C}$ to avoid tissue injury and nociception. ${ }^{17}$

\section{Paradoxical sleep deprivation}

The PSD and the HAB + PSD groups were deprived of rapid eye movement sleep for 23 hours according to the modified multiple platform method. ${ }^{18}$ To prevent the animals from drinking the water while in the apparatus, $60 \mathrm{~mL}$ of fresh lime juice was added to each $2 \mathrm{~L}$ of water. This procedure was important to foster operant behavior in
Table 1 Summary of behavioral testing

\begin{tabular}{lc}
\hline Procedure/behavior/parameter & Process or function \\
\hline $\begin{array}{l}\text { Open field } \\
\text { Spontaneous } \\
\text { Central zone entries }\end{array}$ & \\
Crossing & $\begin{array}{l}\text { Impulsivity/anxiety } \\
\text { Locomotor activity }\end{array}$ \\
Operant conditioning & \\
Goal-directed & \\
Process or function related to $f(L R t)$ & Locomotor activity \\
Scallop pattern & Procedural memory \\
Optimization index & Behavioral efficiency \\
Consummatory & \\
$\mathrm{f}\left(\mathrm{S}^{+}\right)$ & Anhedonia \\
\hline $\mathrm{f}(\mathrm{LRt})=$ all lever response emitted per session; $\mathrm{f}\left(\mathrm{S}^{+}\right)=$amount of
\end{tabular}
obtained and consumed rewards.

the animals, since this concentration of lime juice is sufficient to cause taste aversion in rats. ${ }^{19}$ The water in the tank was completely changed twice per day to avoid insalubrity. During this procedure, the animals were deprived of sleep and water, but not food, since feed pellets were suspended from the perforated roof of the apparatus.

\section{Statistical analysis}

The data were analyzed using SPSS version 21, while GraphPad Prism version 6.0 was used for graph plotting. The Kolmogorov-Smirnov test was used to assess data normality. Locomotor activity (number of crossings) and central zone entries were analyzed with three-way analysis of variance (ANOVA), considering the following factors: time (baseline, short-term, and long-term), PSD exposure (PSD- and PSD+), and HAB exposure (HABand $\mathrm{HAB}+)$. Data from the reinforcement procedure (procedural memory, locomotor activity, efficiency, and anhedonia) were analyzed using a three-way ANOVA and post-hoc Bonferroni test, considering the following factors: time (baseline, short-term, mid-term, and long-term), PSD exposure (PSD- and PSD +), and $\mathrm{HAB}$ exposure $(\mathrm{HAB}-$ and $\mathrm{HAB}+)$. All results are shown as mean \pm standard error of the mean. Significance was set at $95 \%$ with an alpha error of $5 \%(p \leqslant 0.05)$.

\section{Results}

\section{Effects of repeated stress on spontaneous behavior}

Spontaneous locomotor activity (crossings) analysis, shown in Figure 2A, indicated significant two-way interactions between time and PSD $\left(F_{[2,84]}=3.648, p=0.03\right)$, as well as between PSD and $\mathrm{HAB}\left(\mathrm{F}_{[1,84]}=6.647, \mathrm{p}=\right.$ $0.012)$. Post-hoc analysis showed that animals exposed to $\mathrm{HAB}+\mathrm{PSD}$ crossed more frequently during the shortterm evaluation than at baseline $(p=0.029)$, although this effect returned to baseline levels during the long-term evaluation (long- vs. short-term, $p=0.023$ ). On the other hand, exposure to $\mathrm{HAB}$ led to fewer crossings during the long-term evaluation than at baseline ( $p=0.017)$. In the post-hoc analysis of treatment groups, exposure to $\mathrm{HAB}+\mathrm{PSD}$ led to more crossings during the short-term 
A

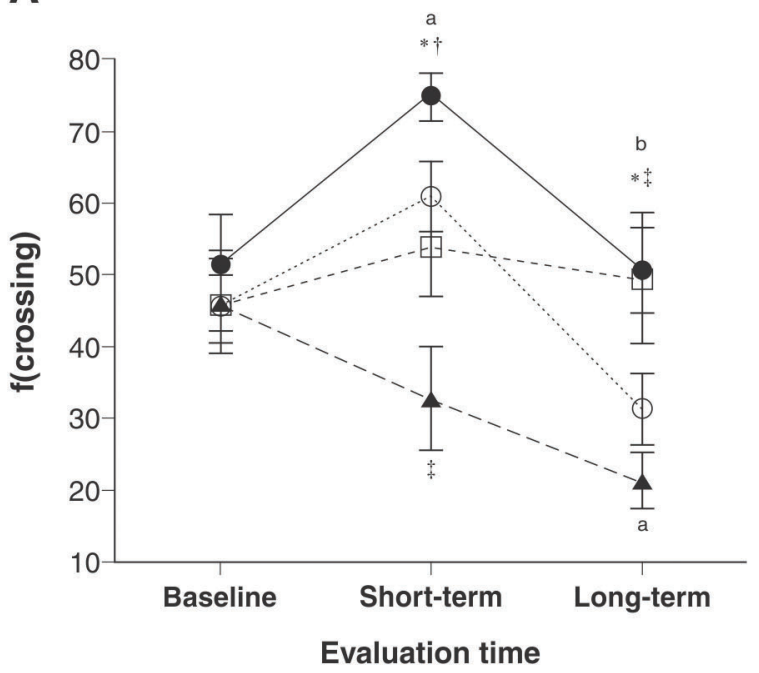

B
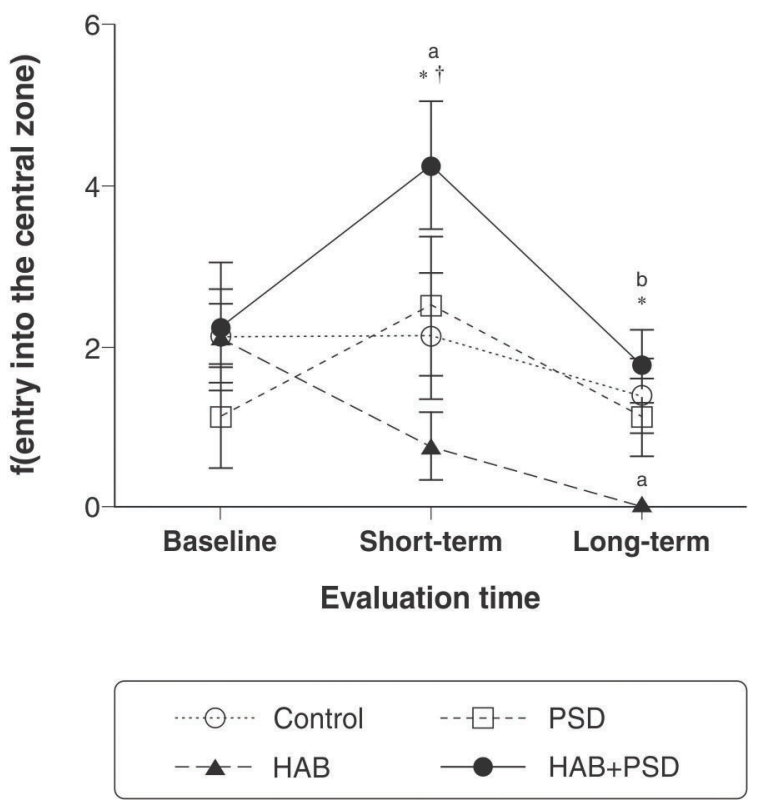

Figure 2 A) Assessment of spontaneous rat locomotor activity (crossings). B) Number of central zone entries at baseline and after short- (1 day) and long-term (5 days) withdrawal from repeated exposure to individual (paradoxical sleep deprivation [PSD], hot air blast [HAB]) or combined environmental stressors (HAB + PSD). Each bar represents mean \pm standard error of the mean of eight animals per group. The specific $p$-values are shown in the results section. ${ }^{a b} p<0.05$ vs. baseline or short-term, respectively. ${ }^{*+\hbar} p<$ 0.05 vs. HAB, PSD, or control, respectively, according to three-way ANOVA with a Bonferroni post-hoc test.

evaluation than exposure to PSD $(p=0.02)$ or HAB alone $(p<0.0001)$. However, at this point there were fewer crossings in the HAB group than in controls $(p=0.036)$. The HAB + PSD group crossed significantly more than the HAB group $(p=0.01)$ or the control group $(p<0.05)$ during the long-term evaluation.
Regarding central zone entries, there were significant two-way interactions between time and PSD $\left(\mathrm{F}_{[2,84]}=\right.$ $3.880, p=0.024)$, as well as between PSD and $\operatorname{HAB}\left(F_{[1,84]}\right.$ $=8.956, p=0.004$ ) (Figure $2 B$ ). Similar to the crossings results, animals exposed to HAB +PSD entered the central zone more frequently in the short-term $(p=$ 0.064), although this returned to baseline levels in the long-term (long- vs. short-term, $p=0.013$ ). On the contrary, during the long-term evaluation, animals exposed to HAB entered the central zone less than at baseline $(p=$ 0.044 ). Post-hoc analysis showed significantly more central zone entries in the HAB + PSD group than in the HAB $(p<0.0001)$ or PSD groups $(p=0.043)$ in the shortterm, while in the long-term, a significant increase was found only in relation to the HAB group $(p=0.043)$.

\section{Effects of repeated stress on goal-directed and consummatory behavior}

Analysis of the $f(L R t)$ (Figure $3 A$ ) indicated a significant three-way interaction between the factors $\left(F_{[3,111]}=3.103\right.$, $p=0.03$ ). Post-hoc analysis showed that, during the short( $p<0.0001)$, mid- $(p<0.0001)$, and long-term $(p<0.0001)$ evaluations, $f(L R t)$ was significantly more frequent in the PSD group than the control group, while at all three points, $f(L R t)$ was less frequent in the HAB + PSD group than the PSD group (short- and mid- $p<0.0001$, long-term $p=$ $0.026)$. In addition, $f(L R t)$ was more frequent in the $H A B$ group than the control group at the mid-term evaluation point $(p<0.05)$.

Regarding $\mathrm{f}\left(\mathrm{S}^{+}\right)$(Figure 3B), there were significant twoway interactions between the time and $\mathrm{HAB}\left(\mathrm{F}_{[3,113]}=8.876\right.$, $p<0.0001)$, as well as between PSD and $\mathrm{HAB}\left(\mathrm{F}_{[1,113]}=\right.$ 13.703, $p<0.0001)$. Lower $f\left(S^{+}\right)$was observed in the HAB + PSD group over time (short-, mid-, and long-term evaluations vs. baseline, $p<0.0001)$, and $f\left(S^{+}\right)$decreased in the HAB group between baseline and the long-term evaluation point $(p=0.021)$. Post-hoc analysis showed that in the short- $(p<0.0001)$, mid- $(p=0.001)$, and long-term $(p=$ $0.005), f\left(S^{+}\right)$decreased significantly in the HAB + PSD group compared to the control group. This decrease was also significant in relation to the PSD group in the short$(p<0.0001)$, mid- $(p<0.0001)$, and long-term $(p<$ $0.0001)$, as well as to the HAB group in the short- $(p<$ $0.0001)$, mid- $(p=0.001)$, and long-term $(p=0.005)$.

According to the optimization index, there was no change in behavioral efficiency over time (Figure 3C).

Finally, Figure 4 shows the effects of repeated stress on cognitive performance (procedural memory) as a discrete measurement of \%LR in the last 30" of FI 1' (scallop pattern). In this analysis, a significant two-way interaction was observed between PSD and $\mathrm{HAB}\left(\mathrm{F}_{[1,111]}=5.393\right.$, $p=0.022)$. In the post-hoc analysis, a disturbance in the scallop pattern was observed over time in the HAB + PSD group (short-term: $p=0.06$; mid-term: $p=0.02$; long-term $p=0.03$ vs. baseline). There was a significant disturbance in the scallop pattern between the HAB + PSD group and the HAB group (mid-term: $p=0.036$; long-term: $p<0.05$ ), the PSD group (short-term: $p=0.008$; mid-term: $p=0.021$; long-term $p=0.02$ ), and the control group (long-term: $p<0.05)$. 
A

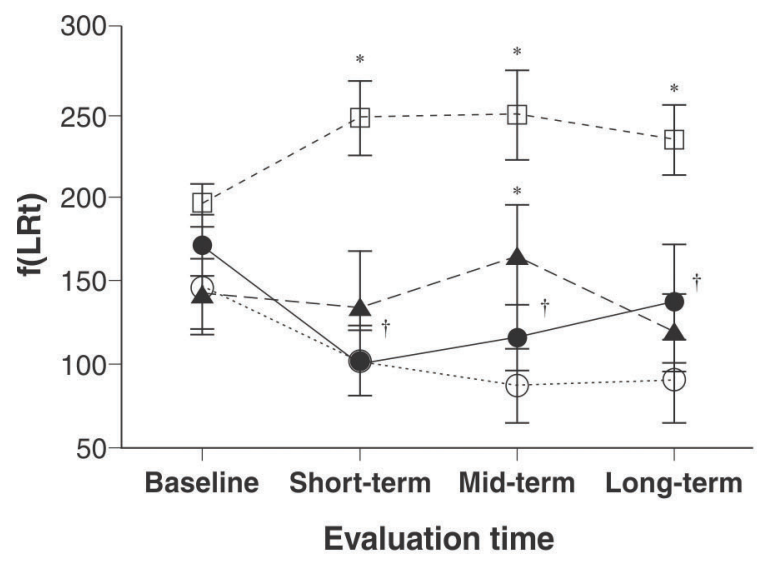

B

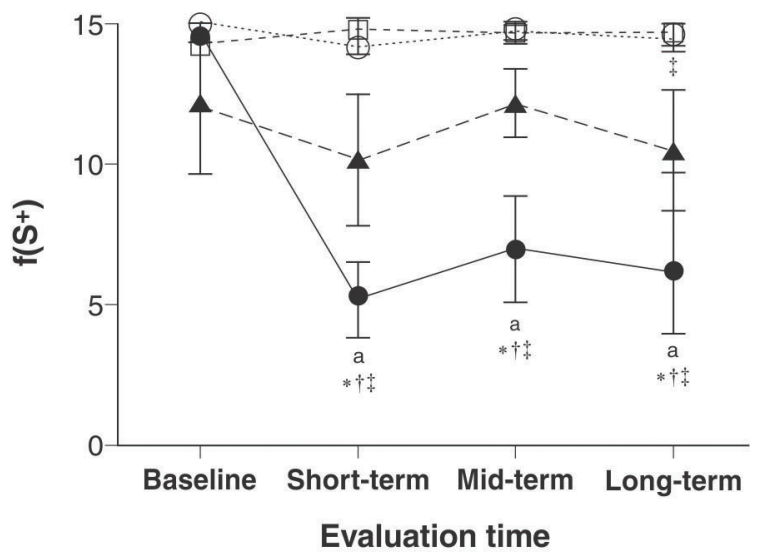

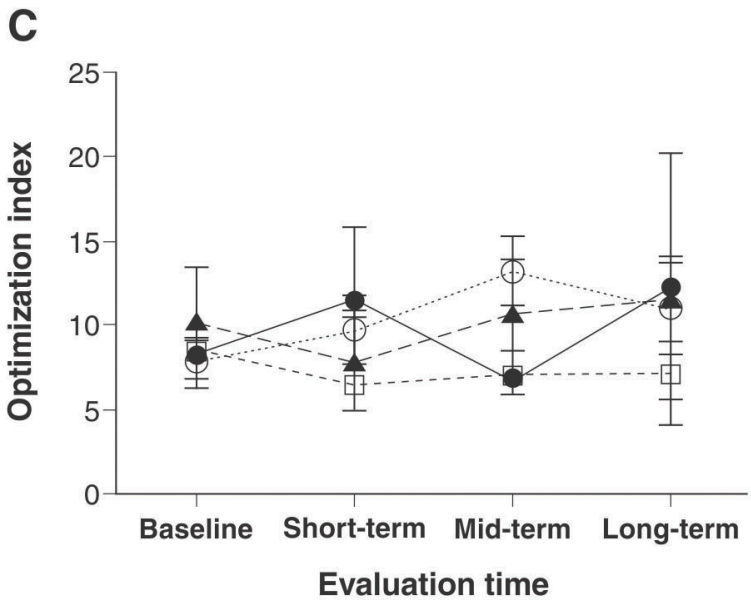

Evaluation time

$\cdots \cdot \odot \cdots$ Control $---7--$ PSD $-₫-\mathrm{HAB} \longrightarrow \mathrm{HAB}+\mathrm{PSD}$

Figure 3 A) Analysis of frequency of lever-press response $f(L R t)$. B) Analysis of frequency of received reward $f\left(S^{+}\right)$. C) Analysis of behavioral efficiency according to baseline optimization index and after short- (1 day), mid- (3 day), and long-term ( 5 day) withdrawal from repeated exposure to individual (paradoxical sleep deprivation [PSD], hot air blast [HAB]) or combined environmental stressors (HAB+PSD). Each bar represents mean \pm standard error of the mean of eight animals per group. The specific $p$-values are shown in the results section. ${ }^{a} p<0.05$ vs. baseline. ${ }^{* \neq} p<0.05$ vs. control, PSD, or HAB, respectively, according to three-way analysis of variance with Bonferroni post-hoc test.

\section{Discussion}

In the present study, we observed that exposing rats to individual or combined environmental stressors (HAB and/or PSD), triggered behavioral alterations similar to endophenotypes of mood and anxiety disorders, depending on the nature of the behavior analyzed.

The first parameter was spontaneous behavior, which was evaluated according to the number of crossings and entries into the central zone of the open field. Spontaneous behavior originates from phylogenetic processes, while LR are goal-directed instrumental behaviors maintained by ontogenetic processes of operant control. ${ }^{20} \mathrm{We}$ observed that repeatedly exposing rats to environmental stressors caused intense and immediate disruptive effects on instrumental locomotor activity. In other words, repeated exposure to $H A B$ induced a progressive decrease in spontaneous activity that was more pronounced in the long term (5 days post-repeated stress). It should be pointed out that some studies have reported that HAB has punitive properties. ${ }^{16}$ On the other hand, at the long-term evaluation point, the PSD group presented operant hyperactivity. Curiously, repeated exposure to both stressors (HAB+PSD) produced spontaneous short-term hyperactivity, short- and mid-term operant hypoactivity, and no long-term changes. This means that $\mathrm{HAB}+\mathrm{PSD}$ caused distinct behavioral alterations depending on the type of behavior evaluated. Although some studies have suggested that HAB and PSD have opposite effects on locomotor activity, we found no 


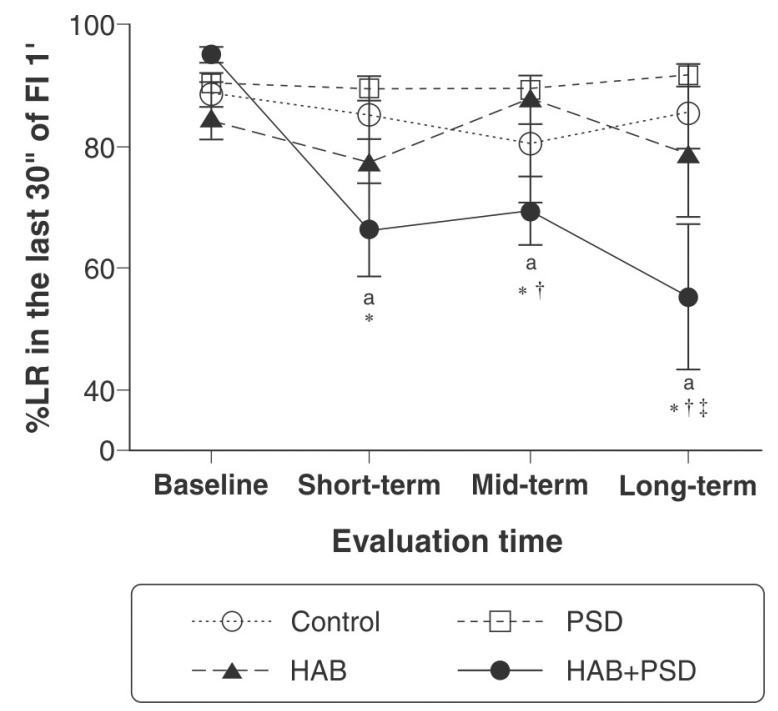

Figure 4 Evaluation of rat procedural memory (scallop pattern) based on the \%LR measure in the last 30" of FI 1' at baseline and after short-term (1 day), mid-term (3 days), and long-term (5 days) withdrawal from repeated exposure to either paradoxical sleep deprivation (PSD), hot air blast (HAB), or a combination of the two stressors (HAB + PSD). Each bar represents mean \pm standard error of the mean of eight animals per group. The specific $p$-values are shown in the results section. ${ }^{a} p<0.05$ vs. baseline. ${ }^{*+1} p<0.05$ vs. $\mathrm{PSD}, \mathrm{HAB}$, or control, respectively, according to three-way analysis of variance with a Bonferroni post-hoc test.

evidence of their individual or combined effects on operant behavior. ${ }^{13,16,21,22}$

At the long-term evaluation point, the control group displayed spontaneous hypoactivity, despite not having been exposed to stressful events. This hypoactivity could have been due to habituation with the open-field test. Indeed, a number of studies have reported such an effect in open-field testing when a test-retest design was used. ${ }^{23,24}$ On the other hand, HAB induced a lower crossing frequency than PSD or $\mathrm{HAB}+\mathrm{PSD}$ at all poststress evaluation points. Since both the control group and the HAB group showed a long-term allostatic load, we suggest that the PSD group also showed long-term spontaneous hyperactivity. ${ }^{6}$

We observed no changes in behavioral efficiency, which was calculated as an optimization index (i.e., operant efficiency). This demonstrates that all groups could perform the tests with no motor impairment.

We also assessed emotional (i.e., anxiety) and motivational (i.e., reward) functions. In our experimental condition, HAB or PSD alone caused opposite impulsivity and anxiety effects, as has been observed in previous studies. ${ }^{13,25,26}$ This could indicate that HAB functioned as an anxiogenic stimulus, since there was an almost complete lack of central zone entries at the long-term evaluation point. Nevertheless, HAB + PSD induced a short-term increase in central zone entries that returned to baseline levels in the long-term. In contrast, PSD did not affect impulsivity/anxiety, despite previous reports to the contrary. ${ }^{27}$ Furthermore, the HAB + PSD group exhibited higher impulsivity than the HAB group in the short and long term. Thus, we can infer that only a combination of two types of repeated stressors can induce some degree of impulsivity, albeit temporarily.

The present study used reward-related consumption, which is typical of a reinforcement model, to evaluate a state of loss of interest. ${ }^{28}$ Our results suggest that relevant short-, mid-, and long-term deficits in reward function (i.e., motivational dysfunction) were caused by exposure to $\mathrm{HAB}+\mathrm{PSD}$, i.e. a $50 \%$ decrease of reward production compared to baseline. This was corroborated by the longterm evaluation results, since the HAB + PSD group differed from the non-stressed control group and the PSD group (mania-like group) but not the HAB group (depressive-like group). Given that the most commonly used test to evaluate motivational changes in rodents is the sucrose preference test, our behavioral evaluation of reward function with a reinforcement model is innovative. ${ }^{29}$

The HAB + PSD group exhibited a persistent deficit in procedural memory at all post-stress evaluation points, despite presenting better performance at baseline. Moreover, the persistent disruptive effects of $\mathrm{HAB}+\mathrm{PSD}$ on instrumental behavior were not observed when the animals were exposed to either stressor individually. However, since all of the evaluated stressors caused great decreases in LR in phase II (data not shown), ,0,31 we can infer that repeated stress may cause changes related to anxiety and mood disorders (Table 2). We also observed that exposure to HAB replicated the hypoactivity and anxiety observed in several preclinical models of depression and anxiety. ${ }^{32}$ Despite the hyperactivity observed in the open-field test after PSD exposure, our study demonstrated (for the first time, we believe) a type of operant behavioral activation not associated with an emotional, motivational, or cognitive deficit. In other words, PSD-exposed rats emitted a number of instrumental responses in this task without additional rewards. ${ }^{33}$ It is important to point out that hyperlocomotion is just one symptom related to mania episodes, although it has been used as unique behavioral phenotype in several animal models of bipolar mania. ${ }^{34}$

We also observed that repeated exposure to $\mathrm{HAB}$ + PSD was more deleterious than repeated exposure to a single stressor. Apparently, exposure to HAB + PSD induced endophenotypes similar to depressive episodes, such as (operant) hypoactivity and motivational dysfunction, as well as those similar to mania episodes, such as (spontaneous) hyperactivity, impulsivity, and cognitive deficit. $^{35,36}$ Therefore, the dysfunction of the reward system seems to be a core alteration related to the neurobiology of mood disorders, since its activation is related to depressive and mania episodes. ${ }^{37}$

In previous studies, animals subjected to PSD showed cognitive impairment in ethopharmacological tests. ${ }^{38,39}$ On the other hand, some reviews found that sleep deprivation can be indicated for treating severely depressed patients. ${ }^{40}$ It should be pointed out, however, that most of these studies did not assess the effects of sleep deprivation on procedural memory related to goal-directed behavior. In fact, 72-hour PSD protocols have been used prior to behavioral evaluations, ${ }^{39,41}$ and $24-$ or 48 -hour 
Table 2 Endophenotypes induced in this protocol and most prevalent related psychiatric disorder

\begin{tabular}{|c|c|c|c|c|}
\hline \multirow[b]{2}{*}{$\begin{array}{l}\text { Stress } \\
\text { modality }\end{array}$} & \multicolumn{3}{|c|}{ Endophenotypes induced } & \multirow[b]{2}{*}{$\begin{array}{l}\text { Related psychiatric } \\
\text { disorder }\end{array}$} \\
\hline & Behavioral & Affective & Cognitive & \\
\hline PSD & Operant hyperactivity & - & - & Manic episode \\
\hline HAB & Spontaneous hypoactivity & Anxiety & - & Depression, anxiety \\
\hline HAB + PSD & $\begin{array}{c}\text { Spontaneous hyperactivity + operant } \\
\text { hypoactivity }\end{array}$ & $\begin{array}{l}\text { Impulsivity } \\
\text { + anhedonia }\end{array}$ & $\begin{array}{l}\text { Procedural memory } \\
\text { impairment }\end{array}$ & Bipolar disorder \\
\hline
\end{tabular}

$\mathrm{HAB}=$ hot air blast; $\mathrm{PSD}=$ paradoxical sleep deprivation.

PSD protocols have been used to investigate memory impairment in ethopharmacological tests. ${ }^{39}$ Thus, some of the divergences between clinical and preclinical data may be due to methodological issues, such as: the different types of memory assessed, training time, type of task, time awake, stage of interrupted sleep, and experimental design type. ${ }^{40}$ Hence, to better address this issue, in the present study five 23-hour PSD sessions were performed on alternate days to assess different behaviors. We think that, in further studies, other behavioral procedures, distinct from those conducted here, could further explain the lack of memory deficit in the PSD group. For instance, quantifying sleep deprivation, maintaining reinforcement throughout the stress exposure phases, the type of behavior assessed (e.g., arbitrary or species-specific), and cognitive function measurement are important features to be evaluated.

Altogether, we think that the memory deficit observed in the HAB + PSD group may be compared to some endophenotypes characteristic of bipolar disorder. Clinical studies suggest that the memory is one of the most impaired cognitive functions in both euthymic and symptomatic patients. On the other hand, deficits in attention and executive control may be related to impulsivity. ${ }^{42}$ Furthermore, the number of mood episodes is associated with cognitive impairment. ${ }^{6}$

The present study has some limitations that should be addressed in future studies related to face, predictive, and construct validities. Face validity (e.g., assessing the effects of forced swimming, sucrose preference, passive avoidance, and Morris water maze tests), predictive validity (e.g., assessing the effects of lithium, valproic acid, or other mood stabilizers), and construct validity (e.g., assessing neurochemical effects) must be carefully addressed. We also did not evaluate the influence of sex in this animal model. Our decision was guided by evidence of an equal gender ratio in bipolar disorder prevalence. ${ }^{43}$ Nevertheless, it has been reported that women have a higher risk of bipolar II/hypomania, rapid cycling, mixed episodes, and comorbidities. ${ }^{44}$ Based on the limitations presented here, we can suggest that our study presents a putative animal model of bipolar disorder.

In conclusion, this study presents a low-cost animal model of bipolar disorder based on environmental manipulation (i.e., repeated exposure to $H A B+P S D$ ) that induces long-term complex behaviors that result in mixed but non-cyclic phenotypes of mood disorders, which extends the face validity of this animal model.
Thus, based on these behavioral evaluations, we agree that stressful environmental events may be interpreted as complex causes that, by interacting with internal processes, trigger neurophysiological endophenotypes related to mood episodes.

\section{Disclosure}

The authors report no conflicts of interest.

\section{References}

1 Whiteford HA, Degenhardt L, Rehm J, Baxter AJ, Ferrari AJ, Erskine $\mathrm{HE}$, et al. Global burden of disease attributable to mental and substance use disorders: findings from the Global Burden of Disease Study 2010. Lancet. 2013;382:1575-86.

2 Joëls M, Baram TZ. The neuro-symphony of stress. Nat Rev Neurosci. 2009;10:459-66.

3 Scharf SH, Schmidt MV. Animal models of stress vulnerability and resilience in translational research. Curr Psychiatry Rep. 2012; 14:159-65.

4 Jaggi AS, Bhatia N, Kumar N, Singh N, Anand P, Dhawan R. A review on animal models for screening potential anti-stress agents. Neurol Sci. 2011;32:993-1005.

5 Flandreau El, Toth M. Animal models of PTSD: a critical review. Curr Top Behav Neurosci. 2018;38:47-68.

6 da Costa SC, Passos IC, Lowri C, Soares JC, Kapczinski F. Refractory bipolar disorder and neuroprogression. Prog Neuropsychopharmacol Biol Psychiatry. 2016;70:103-10.

7 O'Tousa D, Grahame N. Habit formation: implications for alcoholism research. Alcohol. 2014;48:327-35.

8 Catania AC Learning. 5th ed. Cornwall-on-Hudson: Sloan; 2013.

9 Landgraf D, McCarthy MJ, Welsh DK. Circadian clock and stress interactions in the molecular biology of psychiatric disorders. Curr Psychiatry Rep. 2014;16:483.

10 Levenson J, Frank E. Sleep and circadian rhythm abnormalities in the pathophysiology of bipolar disorder. Curr Top Behav Neurosci. 2011; 5:247-62.

11 Yin X, Guven N, Dietis N. Stress-based animal models of depression: do we actually know what we are doing? Brain Res. 2016;1652:30-42.

12 Benedetti F, Fresi F, Maccioni P, Smeraldi E. Behavioural sensitization to repeated sleep deprivation in a mice model of mania. Behav Brain Res. 2008;187:221-7.

13 Myers TM, Cohn SI, Clark MG. Acquisition and performance of twoway shuttlebox avoidance: Effects of aversive air intensity. Learn Motiv. 2005;36:312-21.

14 Boyko M, Kutz R, Grinshpun Y, Zvenigorodsky V, Gruenbaum SE, Gruenbaum BF, et al. Establishment of an animal model of depression contagion. Behav Brain Res. 2015;281:358-63.

15 Tejada J, Chaim KT, Morato S. X-PloRat: a software for scoring animal behavior in enclosed spaces. Psicol Teor Pesq. 2017;33:1-4.

16 Mayer PCM, da Silva GF, Neto MBC. Punishment of instrumental and consummatory responses with a hot air blast in rats. Behav Anal Res Pract. 2015;15:58-64.

17 Casarrubea M, Sorbera F, Santangelo A, Crescimanno G. The effects of diazepam on the behavioral structure of the rat's response 
to pain in the hot-plate test: anxiolysis vs. pain modulation. Neuropharmacology. 2012;63:310-21.

18 Suchecki D, Duarte Palma B, Tufik S. Sleep rebound in animals deprived of paradoxical sleep by the modified multiple platform method. Brain Res. 2000;875:14-22.

19 Myers KP, Sclafani A. Conditioned acceptance and preference but not altered taste reactivity responses to bitter and sour flavors paired with intragastric glucose infusion. Physiol Behav. 2003; 78:173-83.

20 Pierce DW, Cheney C. Behavior analysis and learning. 3rd ed. New Jersey: Lawrence Erlbaum Associates; 2004.

21 Kanazawa LK, Vecchia DD, Wendler EM, Hocayen PA, dos Reis Lívero FA, Stipp MC, et al. Quercetin reduces manic-like behavior and brain oxidative stress induced by paradoxical sleep deprivation in mice. Free Radic Biol Med. 2016;99:79-86.

22 Davis CJ, Taishi P, Honn KA, Koberstein JN, Krueger JM. P2X7 receptors in body temperature, locomotor activity, and brain mRNA and IncRNA responses to sleep deprivation. Am J Physiol Regul Integr Comp Physiol. 2016;311:R1004-12.

23 Klejbor I, Ludkiewicz B, Turlejski K. Effect of light-dark changes on the locomotor activity in open field in adult rats and opossums. Folia Morphol (Warsz). 2013;72:300-5.

24 Saraceno GE, Caceres LG, Guelman LR, Castilla R, Udovin LD, Ellisman $\mathrm{MH}$, et al. Consequences of excessive plasticity in the hippocampus induced by perinatal asphyxia. Exp Neurol. 2016;286:116-23.

25 Yin M, Chen Y, Zheng H, Pu T, Marshall C, Wu T, et al. Assessment of mouse cognitive and anxiety-like behaviors and hippocampal inflammation following a repeated and intermittent paradoxical sleep deprivation procedure. Behav Brain Res. 2017;321:69-78.

26 Kamphuis J, Baichel S, Lancel M, de Boer SF, Koolhaas JM, Meerlo $P$. Sleep restriction in rats leads to changes in operant behaviour indicative of reduced prefrontal cortex function. J Sleep Res. 2017; 26:5-13.

27 Demos KE, Hart CN, Sweet LH, Mailloux KA, Trautvetter J, Williams $\mathrm{SE}$, et al. Partial sleep deprivation impacts impulsive action but not impulsive decision-making. Physiol Behav. 2016;164:214-9.

28 Xu P, Wang K, Lu C, Dong L, Chen Y, Wang Q, et al. Effects of the chronic restraint stress induced depression on reward-related learning in rats. Behav Brain Res. 2017;321:185-92.

29 Malkesman O, Austin DR, Chen G, Manji HK. Reverse translational strategies for developing animal models of bipolar disorder. Dis Model Mech. 2009;2:238-45.

30 Borquez M, Contreras MP, Vivaldi E, Born J, Inostroza M. Postlearning sleep transiently boosts context specific operant extinction memory. Front Behav Neurosci. 2017;11:74.
31 do Nascimento GS, Monteiro PCM, Gouveia A Jr, Carvalho Neto MB. Subchronic effects of fluoxetine on conditioned suppression produced by a hot air blast. Psychol Neurosci. 2012;5:117-22.

32 Ebner K, Singewald N. Individual differences in stress susceptibility and stress inhibitory mechanisms. Curr Opin Behav Sci. 2017;14:54-64.

33 Mace FC, McComas JJ, Mauro BC, Progar PR, Taylor B, Ervin R, et al. Differential reinforcement of alternative behavior increases resistance to extinction: clinical demonstration, animal modeling, and clinical test of one solution. J Exp Anal Behav. 2010;93:349-67.

34 Le-Niculescu H, McFarland MJ, Ogden CA, Balaraman Y, Patel S, Tan J, et al. Phenomic, convergent functional genomic, and biomarker studies in a stress-reactive genetic animal model of bipolar disorder and co-morbid alcoholism. Am J Med Genet Part B Neuropsychiatr Genet. 2008;147B:134-66.

35 Logan RW, McClung CA. Animal models of bipolar mania: the past, present and future. Neuroscience. 2016;321:163-88.

36 Kato T, Kasahara T, Kubota-Sakashita M, Kato TM, Nakajima K Animal models of recurrent or bipolar depression. Neuroscience. 2016;321:189-96.

37 Nusslock R, Almeida JR, Forbes EE, Versace A, Frank E, Labarbara $\mathrm{EJ}$, et al. Waiting to win: elevated striatal and orbitofrontal cortical activity during reward anticipation in euthymic bipolar disorder adults. Bipolar Disord. 2012;14:249-60.

38 Godoi FR, Oliveira MG, Tufik S. Effects of paradoxical sleep deprivation on the performance of rats in a model of visual attention. Behav Brain Res. 2005;165:138-45.

39 Wadhwa M, Kumari P, Chauhan G, Roy K, Alam S, Kishore K, et al. Sleep deprivation induces spatial memory impairment by altered hippocampus neuroinflammatory responses and glial cells activation in rats. J Neuroimmunol. 2017;312:38-48.

40 Alkadhi K, Zagaar M, Alhaider I, Salim S, Aleisa A. Neurobiological consequences of sleep deprivation. Curr Neuropharmacol. 2013;11: 231-49.

41 Ruiz FS, Andersen ML, Guindalini C, Araujo LP, Lopes JD, Tufik S. Sleep influences the immune response and the rejection process alters sleep pattern: evidence from a skin allograft model in mice. Brain Behav Immun. 2017;61:274-88.

42 Bora E. Developmental trajectory of cognitive impairment in bipolar disorder: comparison with schizophrenia. Eur Neuropsychopharmacol. 2015;25:158-68.

43 Diflorio A, Jones I. Is sex important? Gender differences in bipolar disorder. Int Rev Psychiatry. 2010;22:437-52.

44 Miller LJ, Ghadiali NY, Larusso EM, Wahlen KJ, Avni-Barron O, Mittal $\mathrm{L}$, et al. Bipolar disorder in women. Health Care Women Int. 2015; 36:475-98. 